\title{
Detecting Soil Moisture Content Using Self Balancing Rover
}

\author{
Harisudha Kuresan ${ }^{1}$, Abhinav Rajput ${ }^{2}$
}

\begin{abstract}
The depleting health of the soil is because of the depreciating water level continuously and is now the major concern in this modern era. This paper focuses on determining the soil moisture content in the soil by the means of help of an automatic balancing robotic system. The main objective of this paper is to develop a robotic system which moves through uneven land without tumbling or rolling over, can be easily controlled or maneuvered through rough land patches and even records data simultaneously to detect the moisture content of the soil at a particular place. The setup is such that the rover is moved through fields and then at a certain interval of time it inserts sensor electrodes in the soil and provides with the precisely accurate readings. This method will be useful for irrigation purposes. As, the farmer can focus more on that part of land and irrigating that patch which has low level of moisture content. The aim of the paper is to determine that this method can decrease the use of water for irrigation purposes.
\end{abstract}

Key words: FDR,GSM,SMS,TDR

\section{INTRODUCTION}

A notable modern systems for soil moisture measurements exploit two properties of soils:- 1-moisture content and 2 -water potential. For measuring these properties of the soil, one has to use the sensors. The cost and efficiency of the sensors is always in question as, it either is not effective, or is very costly. So here we are using xcluma soil moisture meter, which records soil humidity, water proportion using arduino raspberry. We will obtain a real time data information on the infotainment screen. A person can have a predetermined list of minimum and maximum, of the expected or required moisture content that should A notable modern systems for soil moisture measurements exploit two properties of soils:1-moisture content and 2-water potential. For measuring these properties of the soil, one has to use the sensors. The cost and efficiency of the sensors is always in question as, it either is not effective, or is very costly. So here we are using xcluma soil moisture meter, which records soil humidity, water proportion using arduino raspberry. We will obtain a real time data information on the infotainment screen. A person can have a predetermined list of minimum and maximum, of the expected or required moisture content that should be present at that place according to the needs of the crop being irrigated. As the amount of water needed for every crop rotation is different. For example, the water requirement for 'sugarcane' crop is as high as 1800-3000 L for a kilo of sugarcane, whereas, on the other hand, the crop like of 'soybean' only require only $450-500 \mathrm{~mL}$ of water for their entire growth period. So, accordingly, we can also manage the water distribution across field during different crop season and requirement. As we have real time display of data on the screen, the farmer or the user can mark the portions of the fields according to the moisture content being displayed, and can also determine the proportion need of the patch of land by matching the necessity and availability with predetermined values. For dynamically monitoring the soil water content in the farmland, we require a sensor which can rapidly respond, with low delay value, and that too with high accuracy. Some of the more desired features expected are low energy consumption and low effective cost.

\section{EXISTING MODEL}

Some years before the technique used for this purpose was a plaster sensor and tensiometer, but as compared to the present time advancement and technology, their response was unsatisfactory, unreliable and even there were hysteresis issues, like error between wetting and drying. In later time, many dielectric sensors based on TDR and FDR principles were developed. Both the techniques though required only a fraction of seconds, but had their own limitations:- 1) The power supply by underground cables in farmlands is impractical and in large lands very costly and also require a lot of maintenance, and the battery life is very limited. The energy consumption, environmental conditions and human labour cost add to the list of limited use. 2) The transmission of data through data cables for long distance is unrealistic in a farmland, so the other need was to transmit the data wirelessly with data loggers or routers. The probe of the sensor is usually inserted in to soil for reading the moisture level, which is then transferred to a device to form a wireless sensor network. One such device is a soil moisture data logger, in this, digital screen is used to display moisture content readings. Two means of communication can be used for data transmission between the far user and the system :1)the readings using GSM network are sent via Short Messaging Service (SMS) 2)for storage or analysis of readings, it can be stored in the memory card too, which can be easily accessible.

Revised Manuscript Received on July 05, 2019.

Harisudha Kuresan, Department of ECE,

SRMIST, +917397459209,sudhakuresan@gmail.com

Abhinav Rajput, Department of ECE, SRM Institute of

Science and Technology, 603203, India. 


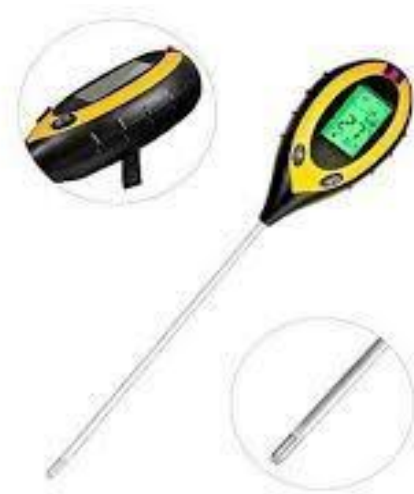

Figure1: A typical soil moisture detector

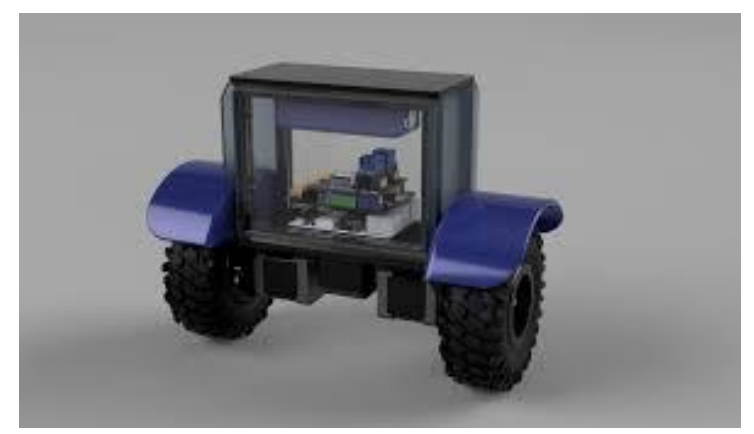

Figure 2: A self balancing rover

\section{PROPOSED METHODOLOGY}

In this upon a rover a sensor is mounted which is automatically balanced, so that it is more efficient in the uneven agricultural grounds and do not tumble and flip over, but maintain its gesture intact. As the rover is controlled by a joystick or can even be programmed to be maneuvered with the help of mobile. The person standing at one corner of the field, can easily move the rover to different parts of the field and take the readings. The sensor system is mounted upon the rover, as the rover moves through the field, it presses the moisture sensor into the soil and thus taking the reading and presenting them on the screen in real time. The rover is powered either by battery or solar panel depending upon the financial asset the farmer or the user wishes to spend. This rover is controlled by a smartphone device or a transmitter.It uses data from the accelerometer and gyroscope to correct its orientation and position. To keep the rover from falling, it must counteract the rover inclination. This action requires feedback and correcting elements. The current orientation of the rover is provided by the micro server. The correcting element is the wheel and motor combination. The rover consist of PID and LQR controllers, of which the main purpose is to prevent the system from falling in forward or backwards movement, whereas the other subsystem of yaw rotation helps in steering and angular regulation when it takes a left or right turn. The rover is composed of stepper motor, which is a DC motor and moves in steps which are discrete. The motor has coil with multiple phase, so when loaded with power it takes a baby step i.e. one step at a time. To power this motor we can harness the solar energy from the sun or can even rotate the motor with the help of mounted battery. The gyro sensor and accelerometer have very efficient and most important work in this simulation. The stabilization by angular velocity is provided with the help of gyro sensor, while, the acceleration forces and balance maintaining, with respect to acceleration and speed are maintained with the help of accelerometer. All the systems and sensors are controlled and maintained in sequence with each other so as to get the maximum efficient output with the help of an arduino board. Arduino can easily read the input signal and can also translate it into output signal, and hence coordinating all the parts together and rotating the motor. We will also mount the rover with the bluetooth module. The bluetooth module will be used to control or maneuver the rover from a distance through mobile or even a joystick. The range of bluetooth module can vary accordingly to the number of modules already present in the market with varying specifications. The use of bluetooth connection is of no cost after once installed with its initial cost, hence making it cost efficient. After reviewing each of the sensor systems on the basis of advantages and disadvantages, it is concluded that selecting soil moisture sensor requires detailed analysis and consideration of many factors, and hence, not an easy task. Even the cost of the system for making it environmental and pocket friendly is also an important factor in this process apart from advantages and disadvantages. However, one must also consider among all the weather factors like temperature, rainfall, type of soil, crop pattern, availability of irrigation options and location of use are all particularly relevant for system use.

\section{CONCLUSION AND FUTURE SCOPE}

The main initiative of this paper is to design a very simple system and sophisticated soil moisture sensor system, by the use of affordable and cheap components that may even provide accurate and reliable readings. The system we intend to develop is primarily based on the agricultural activities. The scope of error and numerous disadvantages have limited the use of previously developed applications which are there in the market. The applications that provide data in suitable form require sophisticated electronic equipment and so are very costly. So we needed an application that could overcome all these limitations and make a leading leap in the marketing prospectus. This soil moisture sensor mounted on a self balancing rover has numerous scope for development and modification. We can always work on increasing the efficiency of the soil sensor with better and long distance relay of information and data and decreasing the delay time in the process. The battery life of the rover can be advanced by doing some more research and increase the effectiveness and even time period of its use. Foreseeing the conditions and looking up ahead on the reports of water level depletion and scarcity of available water, this product will be a game changer and a boom in the market with its capacity and ability to maneuver through rough terrain. On a large application basis, if equipped with very efficient hydraulic and drilling system, then this setup can also be used for measuring the depth of groundwater or for checking the depth at which the adequate water content is present. 


\section{REFERENCES}

V. [1] H.ELLER, "A CAPACITIVE SOIL MOISTURE SENSOR", JOURNAL OF HYDROLOGY, VOLUME-185 [2] T.J.DEAN, "SOIL MOISTURE MEASUREMENT BY AN IMPROVED CAPACITANCE TECHNIQUE, PART I. SENSOR DESIGN AND PERFORMANCE", JOURNAL OF HYDROLOGY, VOLUME-93

VI. [2] ANCHIT GARG, PRIYAMITRA MUNOTH, ROHIT Goyal,"ApPliCATION OF SOIL MOISTURE SENSORS IN AGRICUlTURE:A REVIEW", INTERNATIONAL CONFERENCE ON HYDRAULICS, WATER RESOURCES AND COASTAL ENGINEERING (HydRo 2016), AT PUNE, INDIA, VOLUME: 21

[3] Kartik Madhira, Ammar Gandhi, Aneesha Gujral, "Self balancing robot using complementary filter: Implementation and analysis of complementary filter on SBR", Conference: 2016 International Conference on Electrical, Electronics, and Optimization Techniques (ICEEOT), DOI: 10.1109/ICEEOT.2016.7755240

[4] Gregor O. Novak,"Simple Path Planning Algorithm for Two-Wheeled Differentially Driven (2WDD) Soccer Robots", Conference: Proceedings of the Second Workshop on Intelligent Solutions in Embedded Systems, WISES 2004, Graz University of Technology, Graz, Austria, 2004, June 25

[5] Anton I. Glushchenko, Vladislav A. Petrov, Konstantin A. Lastochkin, "On Development of Neural Network Controller with Online Training to Control Two-Wheeled Balancing Robot", Conference: 2018 International Russian Automation Conference (RusAutoCon), DOI:10.1109/RUSAUTOCON.2018.8501692

[6] Tianyu Liu, Xuhan Wang, Huixinz Zhou, Xunea Che, Huanbao Liu, Qingsheng Wang, "Design and Control of a Two-wheeled Self-balancing Robot made in 3D Printing", Conference: 2018 Chinese Automation Congress (CAC) DOI: 10.1109/CAC.2018.8623557

[7] Chao Wang, Jin Huang, Zichang Guo, Yaguang Wang,'Research of Two-Wheeled Self-Balanced Robot's Disturbance Rejection Control on Uneven Pavement", Conference: 2018 IEEE International Conference on Mechatronics, Robotics and Automation (ICMRA) DOI:10.1109/ICMRA.2018.8490579

[8]Chundong Wang, Jin Huang, Chao Wang, Rui Wu,'Dynamic Surface Active Disturbance Rejection Control for Two-Wheeled Self-Balancing Robot", Conference: The 3rd International Conference DOI: 10.1145/3265639.3265645

[9]Animesh Chhotray, Manas K. Pradhan, Krishna K. Pandey, Dayal R. Parhi, In book: Proceedings of the International Conference on Signal, Networks, Computing, and Systems DOI: 10.1007/978-81-322-3589-7_9 